\title{
Isolation And Identification of Oral Candida Organism in Precancerous And Cancerous Lesion of Oral Cavity.
}

\author{
Dr. Shweta Chaturvedi, Dr. Preeti Nair,Dr. Shantala R Naik, Dr. Rahul Patel. \\ Peoples College Of Dental Science \&Research Center Bhopal
}

\begin{abstract}
Candida may play a role in the etiopathogenesis of premalignant and malignant lesions. The aim of the study was to isolate and identify Candida species from the oral cavity with HiCrome candida differential agar plate. It is a new chromogenic culture media that allows the isolation and identification of species on the basis of coloured colony formation. This study was carried out in 56 patients that included, 18 cases of OSMF, 18 cases of Squamous cell carcinoma and 18 healthy individuals. Hichrom agar medium can show four different candida species depending on the coloured colonies, like light green colonies are identified as Candida albicans, Pink with a darker mauve center colonies as Candida glabrata, pink with pale borders colonies as Candida krusei, and dark blue, purple diffusion colonies as Candida tropicalis. Our study was able to identify the most common candida species in the diseased group as well in healthy individuals.
\end{abstract}

\section{Introduction}

Precancerous lesion is a morphologically altered tissue in which oral cancer is more likely to occur than in its apparently normal counterpart, for example, leukoplakia, oral submucous fibrosis (OSMF).

In 2005, World Health Organization (WHO) introduced the term "Potentially malignant disorders" to combine the precancerous lesions and conditions together, as it is suggestive of, that not all disorders described under this term may transform into cancer.Leukoplakia is defined as "a white patch or plaque that cannot be characterized clinically or pathologically as any other disease". The factors predisposing to leukoplakia include tobacco smoking and chewing, super added Candida infections, human papilloma virus (HPV) (type 16 and 18) infections, atrophic epithelial lesions, persistent trauma, and electrogalvanic current.Oral submucous fibrosis is a chronic disease of the oral cavity characterized by inflammation and progressive fibrosis of the lamina propria and deeper connective tissues. ${ }^{6}$ Tobacco, lime, betel quid, iron and nutritionaldeficiencies, chronic candidiasis, genetic abnormalities, viral infections, autoimmunity are considered to have either direct effect in causing OSMF or an indirect effect by intervening the immune system which is compromised in OSMF. Oral squamous cell carcinoma [OSCC) accounts for 5\% of all cancers in men and $2 \%$ in women. Candida is one among the etiologic risk factors of OSCC, which is part of normal oral flora. Candida infection may occur probably due to weakening of the immune system.Candida organisms are normal commensals that are found colonizing the oral mucosa frequently. Oral Candida is a "yeast -like opportunistic pathogen". Depending on the host defense mechanisms or local oral micro environment, Candida cantransform from a harmless commensal to pathogenic organism causing oral mucosal infection. The Candida genus is comprised of over 150 species of asporogenous 'yeast-like' fungi. ${ }^{2}$ Among these more important pathogenic species are Candida albicans, Candida tropicalis, Candida parapsilopsis, Candida krusei, Candida lusitaniae and Candida glabrata. Candida albicans is the primary cause of oral candidiasis.Certain strains of Candida albicans are postulated to play a key role in the development of dysplasia. ${ }^{1}$ The hyphal form of this organism extends from the mucosal surface to the deeper epithelial cell layers. They produce carcinogenic compounds, like nitrosamines, $\mathrm{N}$ - nitrosobenzyl methylamines which then bind with DNA to form adducts causing miscoding or irregularities with DNA replication. This results in oncogene formation and initiating cancer development. All these findings suggest that there is a strong association between potentially malignant disorders and Candida organism. There are several studies to support the same correlation.

\section{Materials And Method}

This study was conducted to evaluate the different species of candida in patients with OSMF and oral squamous cell carcinoma. In the study there were total 56 cases out of which 18 was OSMF patients, 18 squamous cell carcinoma and 18 healthy controls.Patients were selected from among those visiting the outpatient department of oral medicine and radiology at PCDS \& RC at Bhopal. Patients was clinically diagnosed and grouped according to the diagnosis as having OSMF and Oral OSCC.

Sample swab was collected from the affected area and placed into sterile test tube. It was immediately transported to the laboratory. At laboratory the incubating loop was $1^{\text {st }}$ sterilised and then well was prepared from patients swab on the culture plate, primary and secondary bands were prepared and then finally transported 
into incubator for $48 \mathrm{hrs}$. final identification was done on the basis of colored colonies formed on the chrome agar.

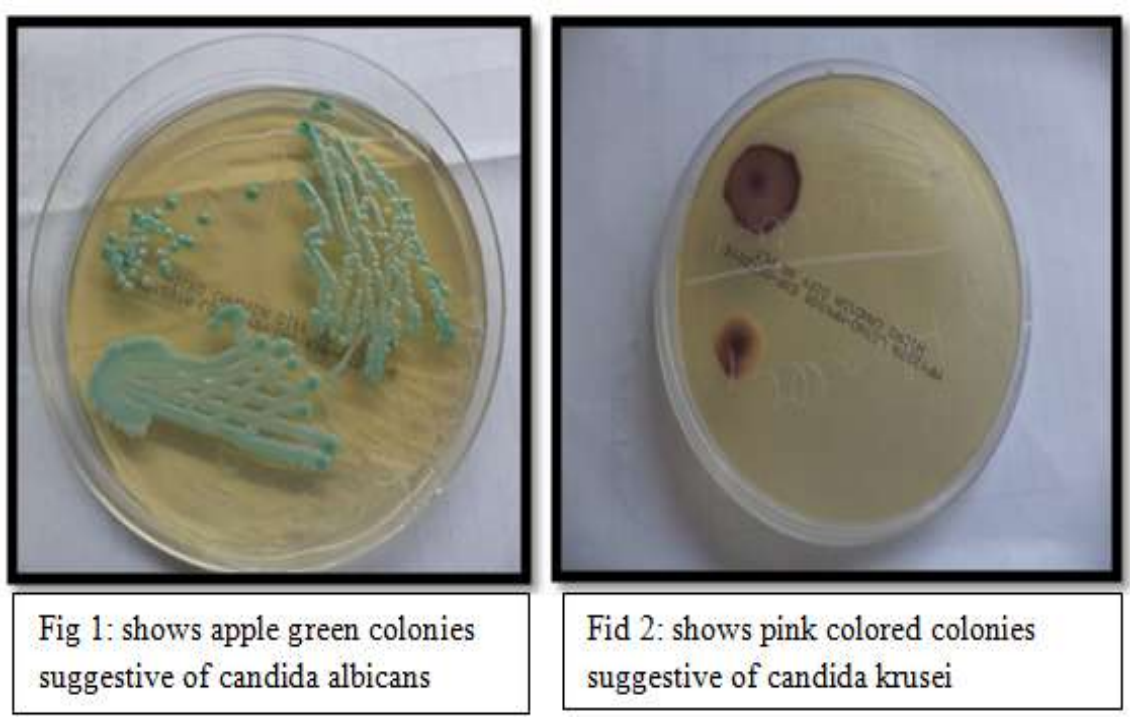

Statistical work-In this study, a chi-squared test was employed to determine the statistical significance of data.

\section{Results}

Demographic distribution of study subjects according to gender(Table $1 \&$ graph 1)

In this study 54 cases were included, out of which 18 cases were of OSMF, out of which 14 were males and 4 were females. Out of 18 cases of OSCC, there were more female patients than males that included 12 females and 6 males.

Mean Age distribution of study subjects (Table $2 \&$ graph 2)

Patients in this study ranged from 17-67 yrs. OSMF patients were in the age range of $2^{\text {nd }}-3^{\text {rd }}$ decade of life (mean age: 27.83yrs). OSCC patients were older in their $6^{\text {th }}$ decade of life (mean age: 54yrs). This distribution was statistically highly significant with a probability value of 0.001 .

Comparison of presence of Candida Species among OSMF, OSCC \& Healthy Control. (Table3\& graph 3)

When the presence of candida species among study groups was compared, the most prevalent candida species was candida albicans (66.7\%) in OSMF patients. In the OSCC group, candida albicans was the most commonly cultured species accounting for $66.7 \%$ followed by candida glabrata $(5.6 \%)$. In healthy individuals the most commonly cultured species was candida albicans, candida glabrata and candida krusei accounting $11.1 \%$ each. These findings were statistically significant with a probability value of 0.008 .

\section{Discussion}

Candida is a yeast- like fungi. Candida is derived from Latin word where toga Candida was a white robe worn by RomanSenators. The most predominant species of candida is the C.albicans which can occur in both yeast and hyphae form. C. albicans has the ability to form the pathogenic commensal which has the ability of colonization, penetration, and damage to host tissues initiation, which could occur due to imbalance in $\mathrm{C}$. albicans virulence factors and host defenses and it may also be due to specific defects in the immune system.

Several other cell surface proteins known as adhesion recognizing host molecules are present. Candida can then produce carcinogenic compounds, such as nitrosamines, $\mathrm{N}$ - nitrosobenzyl methylamine. Strains with high nitrosation potential were isolated from lesions with more advanced precancerous changes. These compounds have ability to bind with DNA to form adductsdue to which the miscoding or irregularities with DNAreplication occurs due to which oncogene formation and cancer initiation occurs. Our study revealed a increased prevalence of candida in OSMF patients $(66.7 \%)$ and also in oral squamous cell carcinoma patients $(66.7 \%)$ when compared to healthy controls $(33.3 \%)$ and these differences were found to be statistically significant $(\mathrm{p}=0.008)$.

These findings were similar to those found by Ariyawardana et al and K. Anila. Study done by Ariyawardana, they found candida albicans in $63.6 \%$ of OSMF cases and 50\% of healthy controls, which is in accordance with our study. This is suggestive of candida albicans prevailing more in OSMF patients than in healthy controls. Study done by K Anila, they reported candida species in $40 \%$ of OSMF cases and $15 \%$ of healthy controls. Also candida albicans was the predominant organism. In this study, most commonly isolated 
species was C.albicans accounting for $66.7 \%$ in OSMF, $66.7 \%$ in oral squamous cell carcinoma were C.albicans and 5.6\% was C.glabrata.Healthy controls, majority $(66.7 \%)$ did not show any growth, however 2 cases each showed C.albicans, C.glabrata and C.krusei.This study showed that candida is more prevalent in OMSF and OSCC. Also the fact that OSCC cases has equal prevalence of candida albicans equal to that of OSMF shows that candida could play a role in the malignant transformation of OSMF to OSCC. We have read reports of OSCC and OSMF going hand and hand. Oral mucosa gets compromised in OSMF which predisposed the individual to candida infection and invasion which supports our study.Also about $21.9 \%$ of candida infected lesions show more severe epithelial dysplasia. So whenever we treat a patient of OSMF we should include topical antifungals in the treatment protocol along with other modality of treatment. Since candida could play a secondary role in the malignant conversion of OSMF cases.

\section{Conclusion}

Our study showed a higher intensity of candida in OSMF and squamous cell carcinoma patients with supporting literature. Candida infected cases of OSMF could have a higher chances of malignant transformation, when it is correlated with candida infected cases of oral squamous cell carcinoma and the degree of dysplasia and prevalence of candida in these dysplastic tissues.

\section{References}

[1]. Sankari SL, Gayathri K, Balachander N, Malathi L. Candida in potentially malignant oral disorders. J Pharm Bioall Sci 2015;7:1624.

[2]. Williams D, Lewis M. Pathogenesis and treatment of oral candidosis. Journal of Oral Microbiology 2011;3:1-11.

[3]. Gupta B, Chandra S, Raj V, Gupta V. Comparison of salivary flow and candidal carriage in patients with oral submucous fibrosis. J Oral Maxillofac Pathol 2015;19:158-63.

[4]. Nair DR, Pruthy R, Pawar U, Chaturvedi P. Oral cancer: Premalignant conditions and screening- an update. Journal of Cancer Research and Therapeutics $2012 ; 8: 57-66$.

[5]. Małgorzata Radwan-Oczko, Magdalena Mendak. Differential diagnosis of oral leukoplakia and lichen planus - on the basis of literature and own observations. J Stoma 2011; 64(5-6): 355-370.

[6]. Sudarshan R, Annigeri RG, Sree Vijayabala G. Pathogenesis of Oral Submucous Fibrosis: The Past and Current Concepts. International Journal of Oral \& Maxillofacial Pathology. 2012;3(2):27-36.

[7]. Jahanahahi G, Shirani S. Detection of Candida albicans in oral squamous cell carcinoma by fluorescence staining technique Dent Res J (Isfahan). 2015 Mar-Apr; 12(2): 115-120.

[8]. Van der Waal I. Oral lichen planus and oral lichenoid lesions; a critical appraisal with emphasis on the diagnostic aspects. Med Oral Patol Oral Cir Bucal. 2009 Jul 1;14 (7):10-4.

[9]. S.Shaista, Gupta.S and Sharma .N. Pathogenecity of candida species in development of oral cancer from pre-cancer in patients of North India population. International Journal of Research in Engineering and Applied Sciences 2016 ;6(2):19-32.

[10]. Galle.F et al Rostello R, Angelillo I F, Ligouri G. Candida spp. in oral cancer and oral precancerous lesions.New Microbiologica 2013;36:283-88.

Table 1: Demographic distribution of study subjects according to gender.

\begin{tabular}{|l|l|l|l|}
\hline Groups & $\begin{array}{l}\text { Male } \\
\mathbf{N}(\%)\end{array}$ & $\begin{array}{l}\text { Female } \\
\mathbf{N}(\%)\end{array}$ & $\begin{array}{l}\text { Total } \\
\mathbf{N}(\%)\end{array}$ \\
\hline OSMF III & $14(25.9 \%)$ & $4(7.4 \%)$ & $18(33.3 \%)$ \\
\hline Squamous Cell Carcinoma & $6(11.1 \%)$ & $12(22.2 \%)$ & $18(33.3)$ \\
\hline Healthy Control & $9(16.7 \%)$ & $9(16.7 \%)$ & $18(33.3 \%)$ \\
\hline Total & $\mathbf{2 9 ( 5 3 . 7 \% )}$ & $\mathbf{2 5}(46.3 \%)$ & 54 \\
\hline Chi Square Value & $\mathbf{7 . 2 9 9}$ & \\
\hline P Value & $\mathbf{0 . 0 2 6 ( S )}$ & \\
\hline
\end{tabular}

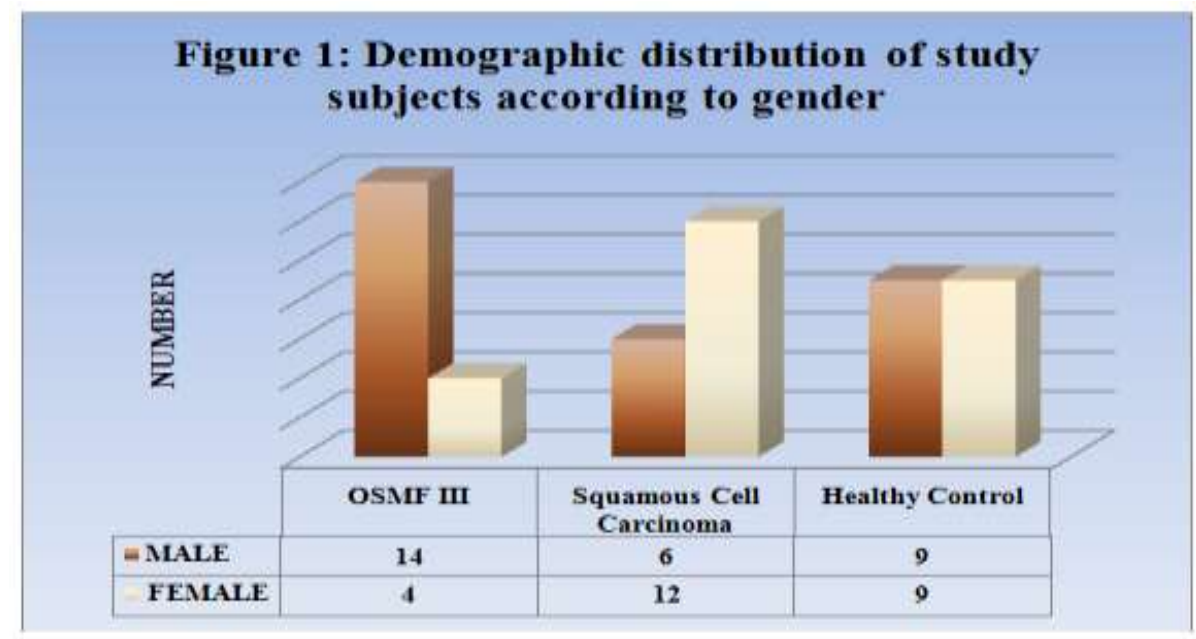


Isolation and Identification of oral candida organism in precancerous and...

Table 2: Mean Age distribution of study subjects

\begin{tabular}{|l|l|l|l|}
\hline Groups & Age (Years) & \multicolumn{1}{l|}{} \\
\hline & Mean & SD & Range \\
\hline OSMF & 27.83 & 6.845 & $17-39$ \\
\hline Squamous Cell Carcinoma & 54.94 & 8.306 & $34-67$ \\
\hline Healthy Control & 30.56 & 9.942 & $19-55$ \\
\hline TOTAL & 37.78 & 14.841 & $17-67$ \\
\hline ANOVA 'F' Value & $\mathbf{5 6 . 0 5 6}$ & \\
\hline P Value & $\mathbf{0 . 0 0 1 ( H S ) ~}$ \\
\hline
\end{tabular}

\section{Figure 2:Mean Age distribution of study subjects}

- Mean Age(Yrs)

\subsection{4}

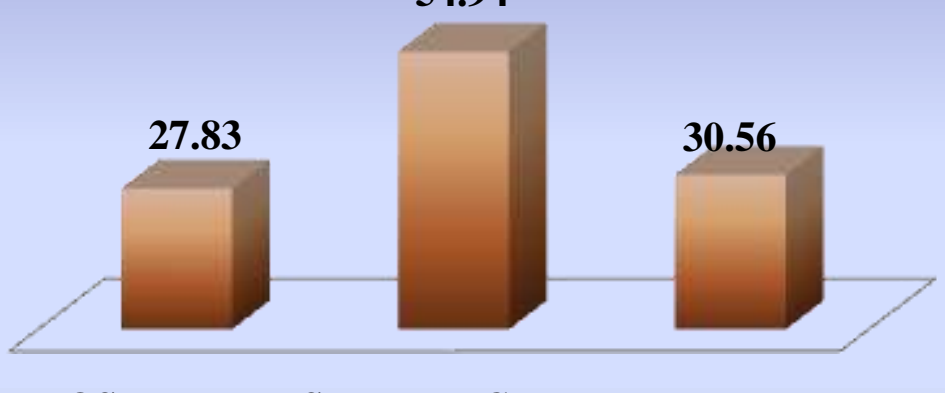

\section{OSMF III Squamous Cell Healthy}

Carcinoma Control

Table 3: Comparison of presence of Candida Species among OSMF III, OSCC \& Healthy Control.

\begin{tabular}{|l|l|l|l|l|}
\hline Groups & Absent & C. Albicans & C. Glabrata & C.Krusi \\
\hline OSMF III & $6(33.3 \%)$ & $12(66.7 \%)$ & $0(0.0 \%)$ & $0(0.0 \%)$ \\
\hline Squamous Cell Carcinoma & $5(27.8 \%)$ & $12(66.7 \%)$ & $1(5.6 \%)$ & $0(0.0 \%)$ \\
\hline Healthy Control & $12(66.7 \%)$ & $2(11.1 \%)$ & $2(11.1 \%)$ & $2(11.1 \%)$ \\
\hline Total & $23(42.6 \%)$ & $26(48.1 \%)$ & $3(5.6 \%)$ & $2(3.7 \%)$ \\
\hline Chi Square Value & $\mathbf{1 7 . 4 3 1}$ & & \\
\hline P Value & $\mathbf{0 . 0 0 8}(\mathbf{S})$ & & \\
\end{tabular}

Figure 3:Comparison of presence of Candida Species among OSMF, OSCC \& Healthy Control.

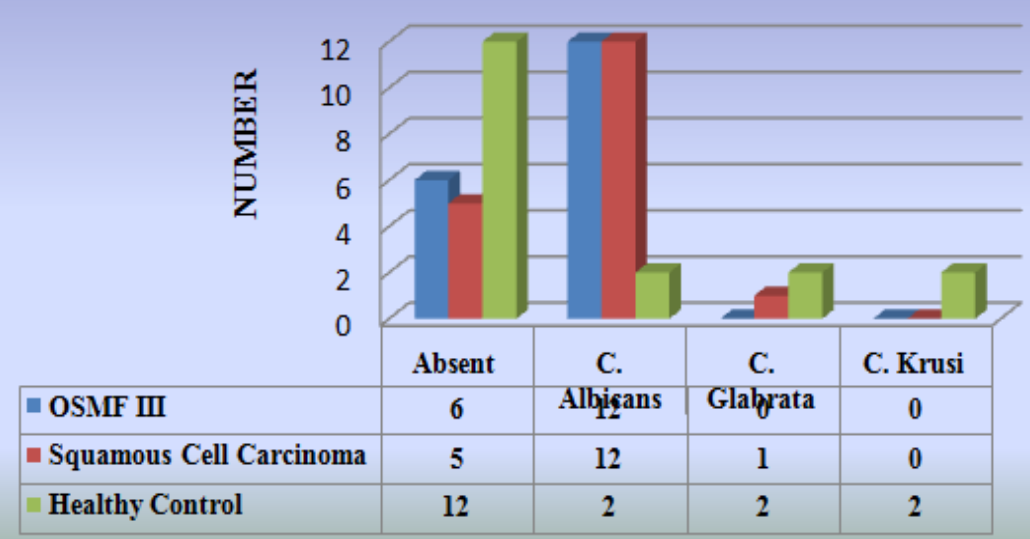

